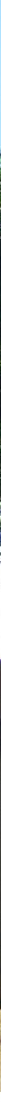

\title{
Vulnerabilidad socioambiental ante fenómenos naturales en las localidades de Texcaltitán, Tonalapan y Nacimiento de Xogapan
}

J.C. Amaro - K. Sieron - F.Córdoba - J. Cervantes

RESUMEN: El municipio de San Andrés Tuxtla se ubica en la costa del Golfo de México (Veracruz) y por sus condiciones climatológicas y topográficas es afectado por distintos fenómenos naturales, principalmente del tipo hidrometeorológico. También, al estar ubicado dentro de Campo Volcánico de Los Tuxtlas, existe la amenaza latente del Volcán San Martín, el cual registró su última erupción en el año de 1793. Adicionalmente, el municipio tiene alta población y altos niveles de marginal lo que incrementa la vulnerabilidad. Desde esta perspectiva, el siguiente trabajo propone la utilización de dos metodologías para obtener los niveles de vulnerabilidad y riesgo, las cuales utilizan métodos cuantitativos, fácilmente replicables y con métodos claros, lo que permitirá su empleo en trabajos futuros. El estudio se aplicó en tres localidades: Nacimiento de Xogapan, Tonalapan y Texcaltitán, obteniendo como resultado altos niveles de vulnerabilidad y peligro, por lo que se consideran necesarias medidas de mitigación.
Palabras Clave: Vulnerabilidad, Peligro, Riesgo, Fenómenos Naturales, Los Tuxtlas.

ABSTRACT: The municipality of San Andrés Tuxtla is located on the coast of the Gulf of Mexico (Veracruz) and due to its climatological and topographic conditions it is affected by different natural phenomena, mainly of hydrometeorological origin. Additionally, due to its location within the Los Tuxtlas Volcanic Field there is the latent threat of the San Martin Volcano, which recorded its last eruption in the year of 1793. Further, the municipality has a high population and high levels of margination, which increases the vulnerability. From this perspective, the following work proposes the use of two methodologies to obtain levels of vulnerability and risk, using quantitative methods easily replicable and with a clear methodology, which allows their use in future work. The study was applied to three locations: Nacimiento de Xogapan, Tonalapan and Texcaltitán, obtaining as a result high levels of 
vulnerability and hazard, for which mitigation measures are considered necessary.

Key words: Vulnerability, Hazard, Risk, Natural Phenomena, Los Tuxtlas

\section{Introducción}

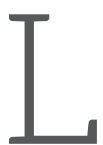

os desastres naturales están presentes en todo el mundo y la situación de vulnerabilidad en el país, ante los fenómenos naturales en general, se presenta en mayor medida en comparación a otras zonas del mundo. Existen varias metodologías para medir la vulnerabilidad de una forma cuantitativa, pero no son replicadas en los estudios analizados. En la amplia bibliografía consultada que incluye Reyes et al. (2013), Lozano (2008), Lara et al.(2006), Hernández et al. (2003), Bolling e Hidajat (2006), entre otros; se constató que, al analizar la vulnerabilidad, diversos estudios hacen a un lado el aspecto social, por lo que se procedió a identificar a la más adecuada y completa para aplicarla a este estudio; se optó por la elaborada por Bohórquez (2013) en la cual propone un índice de vulnerabilidad social para la ciudad de Manzanillo, Colima, utilizando variables que analizan a la vivienda, al individuo, a la localidad, la percepción y a las autoridades, y mediante el análisis de componentes principales propone valores ponderados para cada una de las variables. La ventaja de esta metodología sobre las demás analizadas es su facilidad para ser replicada en otros estudios, incluso a distintas escalas.

San Andrés Tuxtla se ve afectado por distintos fenómenos naturales, entre los que destacan las inundaciones las cuales son provocadas por las fuertes lluvias de hasta 4,500 mm/ anuales en las laderas del Volcán San Martín, propiamente la cabecera municipal y las localidades a estudiar presentan lluvias que van de los 2,000 a los 3,000 mm/anuales (INEGI, 2010), y al tener el municipio una topografía accidentada es común que se presenten diversos tipos de fenómenos relacionados a la inestabilidad de laderas, como son los deslizamientos, derrumbes y flujos a lo largo del año. Por otra parte, la zona de los Tuxtlas se encuentra en una zona de actividad volcánica y en consecuencia existen varias localidades potencialmente amenazadas, principalmente las más cercanas al volcán San Martin al norte de la cabecera municipal.

En este trabajo, se busca obtener los distintos niveles de vulnerabilidad ante fenómenos naturales presentes en las localidades estudiadas y a partir de estos resultados elaborar la cartografía complementaria a los ya existentes mapas de peligro; con la matriz propuesta por Lozano (2008) se realizaron los mapas de riesgo utilizando la fórmula básica:

$$
\text { Riesgo }=\text { Peligro }+ \text { Vulnerabilidad }
$$

Un aspecto a recalcar en este estudio es dar a conocer las diferencias entre los conceptos amenaza, peligro, vulnerabilidad y riesgo, los cuales al ser usados indistintamente terminan siendo confundidos y utilizados como sinónimos; según la definiciones de Chardon y González (2002), una amenaza se refiere a un factor de riesgo, representado por un peligro latente asociado con un fenómeno físico de origen natural o tecnológico, que puede manifestarse en un sitio específico y en un tiempo determinado; produciendo efectos adversos en las personas, los bienes y el medio ambiente, y al peligro lo define como la probabilidad de que un lugar, en un intervalo de tiempo determinado, sea afectado por un evento o fenómeno natural. Mientras al concepto de vulnerabilidad, Wisner et al. (2004) lo define como las características de una persona o grupo y su situación, que influencian su capacidad de anticipar, lidiar, resistir y recuperarse del impacto de una amenaza; y finalmente el concepto riesgo y según la definición de Chardon y González (2002) es la expectación de que algún evento produzca un impacto negativo sobre los elementos antrópicos de un área, si estos elementos no están presentes entonces no existe el riesgo.

\section{Metodología}

Después de recaudar la mayor cantidad po- 
sible de información, tanto de las localidades como las del municipio, incluyendo aspectos del clima, edafología, geología, hidrología y datos sociodemográficos, se planificó el trabajo de campo con la intención de emplear las encuestas proporcionados por Bohórquez (2013) a una cantidad representativa de casas en cada localidad a estudiar. El estudio de Bohórquez (2013) utiliza a las manzanas como objeto de muestra (en la ciudad de Manzanillo), pero en este caso al tener una escala más pequeña, se utilizó a la unidad de vivienda. Para conocer el número de viviendas de cada una de las localidades se procedió a identificarlas mediante percepción remota con el programa Google Earth, ya que este tipo de información actualmente no está disponible (p. ej. INEGI) y a continuación se realizó un muestreo al 13 \% del total de viviendas para determinar cuáles de ellas iban a ser encuestadas, utilizado la herramienta "Random Points" (puntos aleatorios) en el Sistema de Información Geográfico (SIG). Ya teniendo las viviendas seleccionadas, se procedió a realizar las encuestas en las 3 localidades identifi- cadas previamente por autoridades de Protección Civil y Bomberos municipal: Texcaltitán

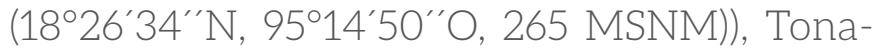
lapan $\left(18^{\circ} 28^{\prime} 08^{\prime \prime} \mathrm{N}, 95^{\circ} 13^{\prime} 12^{\prime \prime} \mathrm{O}, 475 \mathrm{MSNM}\right)$ y Nacimiento de Xogapan (18³1'55”N, 95¹8'20'O, 570 MSNM) (San Andrés Tuxtla) como las más expuestas a fenómenos naturales de diferente índole. La encuesta fue aplicada a cualquier persona mayor de edad que atendiera en la vivienda seleccionada; en el caso de no estar habitada, se encuestó a personas de la vivienda más cercana. La segunda parte del estudio consistió en capturar la información obtenida; en total se aplicaron 128 encuestas (60 en Texcaltitán, 40 en Tonalapan y 28 en Nacimiento de Xogapan). Se utilizaron los valores ponderados de las variables elaborados por Bohórquez (2013) los cuales se muestran en la Tabla 1 y consisten en 2 cuantitativos y 10 cualitativas.

Para obtener IFVS (Índice Final de Vulnerabilidad Social), se agruparon las variables en 4 niveles de exposición, NELP (Nivel de Exposición por Localización), NESC (Nivel de Expo-

\section{Tabla 1. Valores ponderados de variables}

PD Población Dependiente (0.615)

CC Grado de Concentración de la población (0.438)

AA Actividad Antrópica Instalada (0.789)

IFD Ingresos (0.989) (Pesos/Diarios)

NE Nivel de escolaridad (0.989)

IV Informalidad de la vivienda (0.980)

IB Informalidad de la localidad (0.980)

CA Conocimiento de individuo (0.886)

PA Percepción del individuo (0.398)

CH Conocimiento histórico (0.998)

PPC Existencia de planes de prevención (0.997)

RHC Respuesta de la comunidad (0.997)

Tabla 2. Valores ponderados niveles de exposición

\begin{tabular}{|l|l|}
\hline NELP $=0.615 \mathrm{PD}+0.438 \mathrm{CP}+0.789 \mathrm{AA}$ & $\mathrm{NEOI}=0.997 \mathrm{PPC}+0.997 \mathrm{RHC}$ \\
\hline $\mathrm{NESC}=0.989 \mathrm{IFD}+0.989 \mathrm{NE}+0.980 \mathrm{IV}+0.980 \mathrm{IB}$ & $\begin{array}{l}\text { IFVS=0.982 NELP+0.322 NESC+0.927+- } \\
\text { NEEA+0.994 NEOI }\end{array}$ \\
\hline $\mathrm{NEEA}=0.886 \mathrm{CA}+0.398 \mathrm{PA}+0.998 \mathrm{CH}$ & \\
\hline
\end{tabular}

Tabla 3: Valores ponderados Índice Final de Vulnerabilidad Social

IFVS $=0.982 \mathrm{NELP}+0.322 \mathrm{NESC}+0.927$ +NEEA + $0.994 \mathrm{NEOI}$ 
sición con condición Socioeconómica), NEEA (Nivel de Exposición por Empatía con la Amenaza), NEOI (Nivel de Exposición por nivel de Organización Institucional frente a la Amenaza). Al sumar dichos niveles de exposición, se obtiene el IFVS (Tablas 2 y 3 )

Ya obtenidos los valores del IFVS, se crearon cuatro niveles de exposición con los siguientes rangos (Tabla 4).

Con la aplicación de los valores mencionados, se procedió a representar gráficamente los mapas de vulnerabilidad resultantes, utilizando mapas de densidad y poligonización.

\section{Resultados}

Después de establecer las características del nivel de vulnerabilidad (Tabla 4), se tienen los siguientes resultados para cada una de las localidades.

Caso Nacimiento de Xogapan
En Nacimiento de Xogapan, la mayor parte de las viviendas (15) presentan un nivel de vulnerabilidad alto. Esta zona abarca más de la mitad de la localidad (zona sur, este y suroeste); la zona donde se asientan estas viviendas es relativamente más plana comparado con el resto de la localidad. Las viviendas con nivel de vulnerabilidad muy alta son 11, ubicadas en la parte central y norte de la localidad, en zonas con relieve más accidentado. Las viviendas con nivel de vulnerabilidad medio son sólo 2; una de ellas se encuentra en la zona noreste y la otra en el suroeste de la localidad, en zonas relativamente planas (Figura 1, Subfigura A).

\section{Caso Tonalapan}

Tonalapan está dividido en 2: Tonalapan de arriba y Tonalapan de abajo; en ambas partes el nivel de vulnerabilidad predominante es el alto, presente en 21 viviendas, cubriendo casi la totalidad de ambas partes de la localidad, ubicadas en zonas más o menos planas. El nivel de vulnerabilidad muy alto está presente en 8

\section{Tabla 4. Niveles de Exposición}

Baja Vulnerabilidad (Mayor a 15)

Media Vulnerabilidad (Mayor a 12 y menor-igual a 15)

Alta vulnerabilidad (Mayor a 9 y menor-igual a 12)

Muy alta vulnerabilidad (Menor-igual a 9)

Tabla 5. Características de los niveles de vulnerabilidad

\section{Nivel de vulnerabilidad Muy Alto}

Este nivel significa que las viviendas cuentan con las condiciones más precarias, sus habitantes con menores niveles de estudio y bajos ingresos, y el desconocimiento de la amenaza.

\section{Nivel de vulnerabilidad Alto}

En el nivel de vulnerabilidad alto, la mayoría de las viviendas cuentan con techos de lámina y muros de concreto, con niveles de estudio no más altos a la secundaria y con un nivel de conocimiento ante amenazas incipiente.

\section{Nivel de vulnerabilidad medio}

En este caso las viviendas tienen un nivel de informalidad bajo, sus habitantes mejores condiciones de estudios y buenos ingresos, y además de eso cuentan con conocimiento y son conscientes de las amenazas potenciales.

\section{Nivel de vulnerabilidad bajo}

En este nivel, las condiciones de vivienda, estudios e ingresos son buenos, los habitantes están conscientes de las amenazas potenciales, tienen conocimiento histórico y las autoridades logran una buena relación y coordinación con los habitantes de la localidad. 
viviendas, ubicadas en la parte este de Tonalapan de arriba cercana a la ladera y rodeando la parte central de Tonalapan de abajo.

El nivel de vulnerabilidad medio lo presentan sólo en 3 viviendas, 2 en la parte central de Tonalapan de abajo y otra en la parte central de Tonalapan de arriba en una zona relativamente plana (Figura 2, Subfigura A).

\section{Caso Texcaltitán}

En Texcaltitán está asentada entre dos laderas, donde las partes bajas son las de mejor condición económica y en las partes altas viven las personas de escasos recursos. La mayor parte de la localidad tiene un nivel de vulnerabilidad alto; este nivel está presente en viviendas a lo largo del camino principal de la localidad hasta la zona media de la ladera. En total son 31 viviendas encuestadas las que tienen este nivel de vulnerabilidad. Al igual que en Nacimiento de Xogapan, existen viviendas con buenas condiciones estructurales y habitantes con altos niveles de estudios y buenos ingresos, pero que tienen nulo conocimiento sobre los fenómenos y que muestran indiferencia ante las amenazas.

El nivel de vulnerabilidad muy alto se presentó en 24 de las viviendas encuestadas. La mayoría de ellas se ubica en las partes altas de la ladera en donde se asientan las viviendas informales, construidas con materiales precarios; generalmente son recién establecidos, por lo que cuentan con nulo conocimiento histórico sobre amenazas. También en la parte sur de la localidad existen viviendas con alta vulnerabilidad. El nivel de vulnerabilidad medio sólo se presentó en dos viviendas encuestadas, las cuales se ubican en la parte central de la comunidad y cercanas al camino principal, en una zona relativamente plana (Figura 3, Subfigura A). Cabe destacar que, en algunas viviendas, lo que determinó que tuvieran niveles altos de vulnerabilidad, no fueron las condiciones de la vivienda, sino el poco conocimiento y la indiferencia ante las amenazas.

\section{Correlación Vulnerabilidad + Peligro=Riesgo}

Una vez obtenidos los mapas de vulnerabilidad social, se procedió a aprovechar la infor- mación para complementar los mapas de peligro existentes (Sieron et al. 2014), utilizando la matriz elaborada por Instituto de Defensa Civil (INDECI) (tomado del estudio de Lozano, 2008) se elaboraron también mapas de riesgo de cada una de las tres localidades, referentes a caída de ceniza, deslizamientos y lahares.

\section{Peligro}

La localidad de Nacimiento de Xogapan es la más cercana al cráter del volcán San Martin, por lo que presenta un nivel bajo de colapso de techos de concreto por caída de ceniza (CTCCC) y un nivel medio de colapso de techos de lámina por caída de cenizas (CTLCC) en el caso de una nueva erupción; de las otras dos localidades sólo Tonalapan presenta un nivel bajo de peligro de CTLCC. Como se mostró en las secciones anteriores, la zona de Los Tuxtlas presenta grandes cantidades de lluvia durante todo el año, concentrándose principalmente entre los meses de julio a noviembre, lo que hace que las localidades estén propensas al efecto de deslizamientos (y Procesos de Remoción en Masa en general). En las 3 localidades la situación es similar con lluvias anuales promedio mayores a los 2,000 mm y pendientes pronunciadas en varias de ellas INEGI (2010). En lo referente a deslizamientos en las tres localidades existe peligro alto, medio y bajo Figura 1- Subfigura B), Figura 2 -Subfigura B), Figura 3 -Subfigura B).

\section{Riesgo}

Utilizando la matriz propuesta por Lozano (2008) presentada en la Tabla 1, se correlacionaron los mapas de vulnerabilidad elaborados en este estudio con los ya existentes de peligro. Cabe destacar que esta matriz (Tabla 6) fue la que mejor se prestó para ser aplicada, además de ser muy sencilla.

Gracias a la matriz mencionada se obtuvo lo siguiente: Figura 1-Subfigura C). Figura 2-Subfigura C) y Figura 3-Subfigura C).

Propuesta de medidas de mitigación de vulnerabilidad

Con la información obtenida de las encuestas, se concluyó que existen dos variables que determinan en mayor medida los niveles de la 
vulnerabilidad de las viviendas: existencia de planes de prevención y respuesta de la comunidad; ambas variables obtuvieron los mismos resultados en las tres localidades por lo que se optó por crear distintos escenarios con dichas variables y así observar cómo influyen en los niveles de vulnerabilidad.

En el caso de existencia de planes de prevención (en el cual existen 3 valores: 1 "Inexistente", 2 "Incipiente" y 3 "Suficiente"), se determinó que en las localidades, los planes de prevención existen, pero las opiniones tanto de Protección Civil como de los habitantes se contradicen, lo que hace entender que dichos planes no están funcionando como deberían, así que en el estudio se le asignó el valor 2 de "Incipiente" a las 3 localidades. Si el valor fuera el nivel 3 "suficiente" los niveles de vulnerabilidad serían menores, como por ejemplo, en Nacimiento de Xogapan en vez de tener 2 viviendas con vul-

Tabla 6: Matriz de correlación de niveles de peligro y vulnerabilidad

\begin{tabular}{|c|c|c|c|c|c|c|c|c|c|c|}
\hline & & & \multicolumn{8}{|c|}{ NIVELES DE VULNERABILIDAD } \\
\hline & & & \multicolumn{2}{|c|}{ MUY ALTO } & \multicolumn{2}{|r|}{ ALTO } & \multicolumn{2}{|c|}{ MEDIO } & \multicolumn{2}{|c|}{ BAJO } \\
\hline & & & \multicolumn{2}{|r|}{4} & \multicolumn{2}{|r|}{3} & \multicolumn{2}{|r|}{2} & \multicolumn{2}{|r|}{1} \\
\hline \multirow{4}{*}{$\begin{array}{l}\text { NIVELES DE } \\
\text { PELIGRO }\end{array}$} & MUY ALTO & 4 & 4 & Muy Alto & 4 & Muy Alto & 3 & Alto & 3 & Alto \\
\hline & ALTO & 3 & 4 & Muy Alto & 3 & Alto & 2 & Medio & 2 & Medio \\
\hline & MEDIO & 2 & 3 & Alto & 2 & Medio & 2 & Medio & 1 & Bajo \\
\hline & BAJO & 1 & 3 & Alto & 2 & Medio & 1 & Bajo & 1 & Bajo \\
\hline
\end{tabular}

Figura 1: Mapas de vulnerabilidad socioambiental, peligro de deslizamiento por lluvias y riesgo de la localidad de Nacimiento de Xogapan

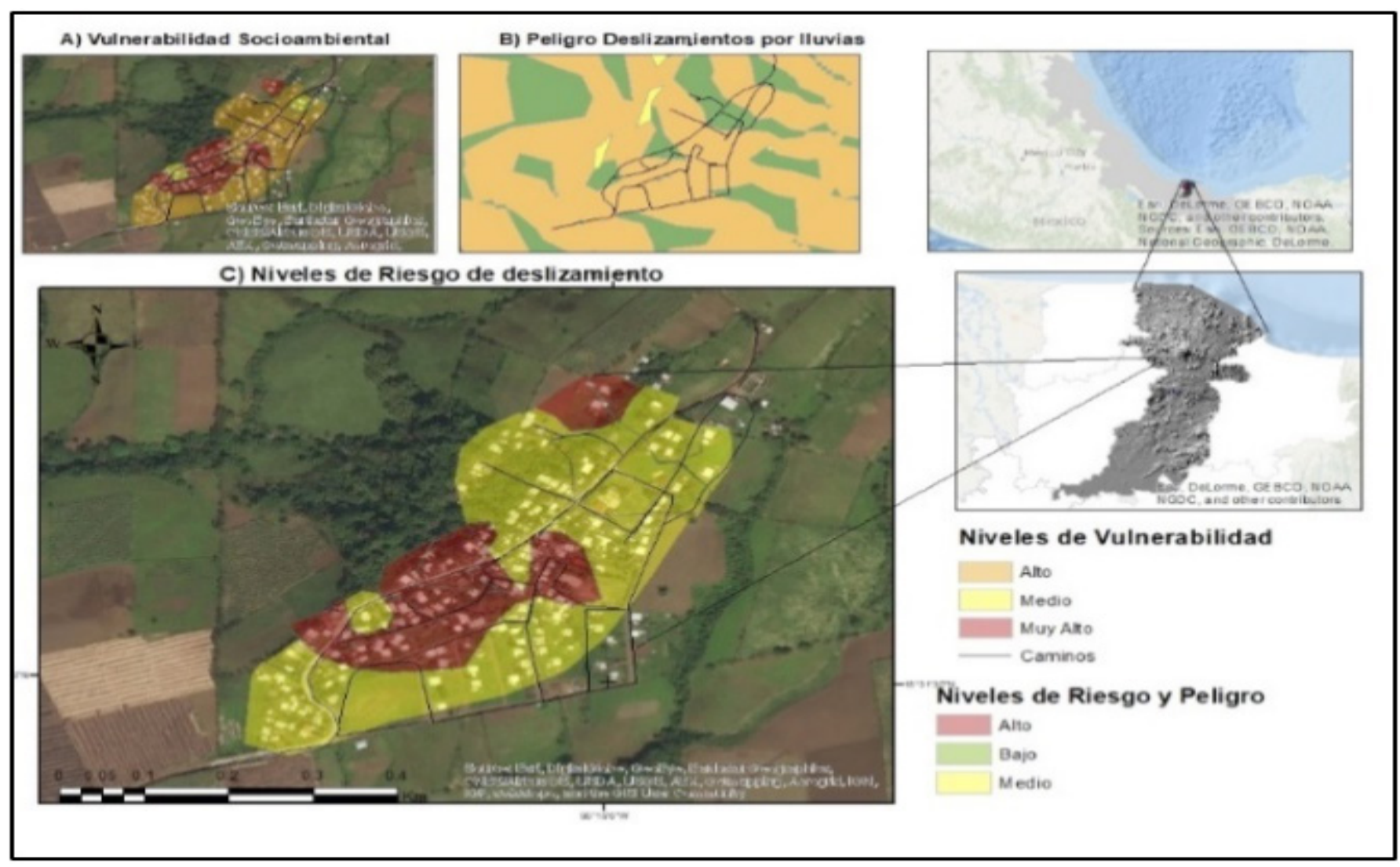


Figura 2: Mapas de vulnerabilidad socioambiental, Peligro de deslizamiento por lluvias y riesgo de la localidad de Tonalapan
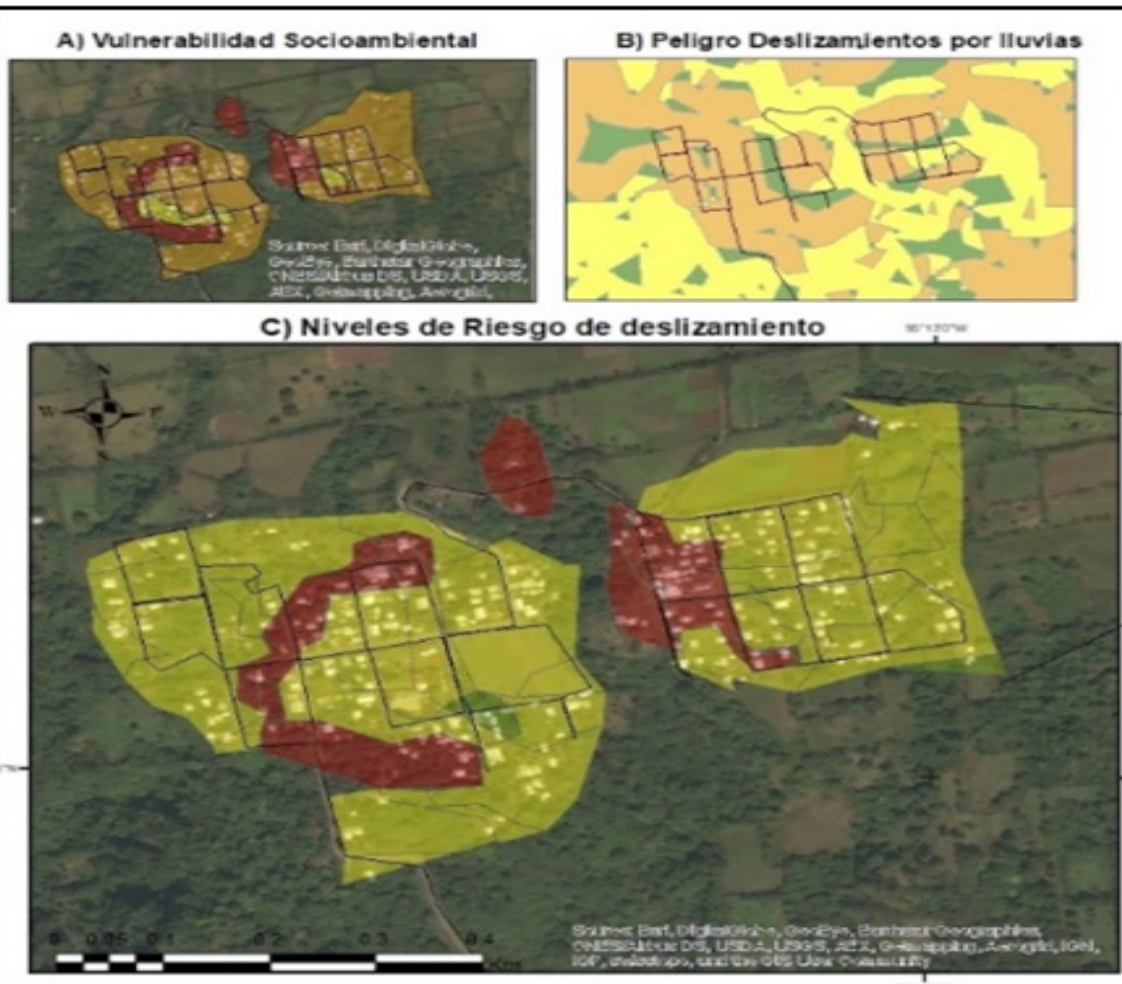

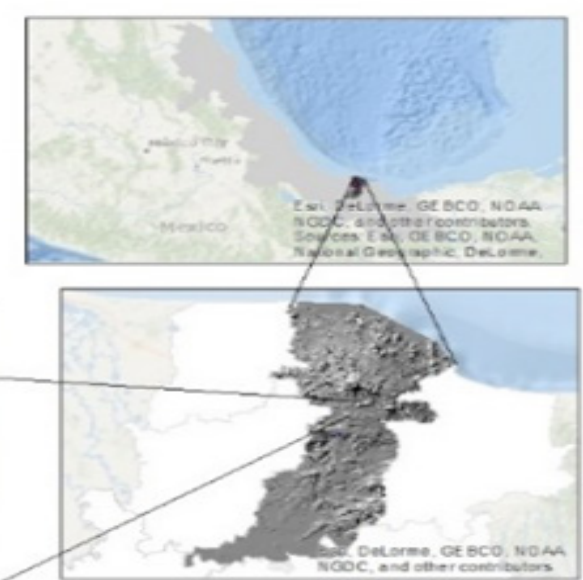

Niveles de Vulnerabilidad

Alto

Medio

Muy ARO

- Camino:

Niveles de Riesgo y Peligro

Ninto

Bajo

Medio

Figura 3: Mapas de vulnerabilidad socioambiental, peligro de deslizamiento por lluvias y riesgo de la localidad de Texcaltitán

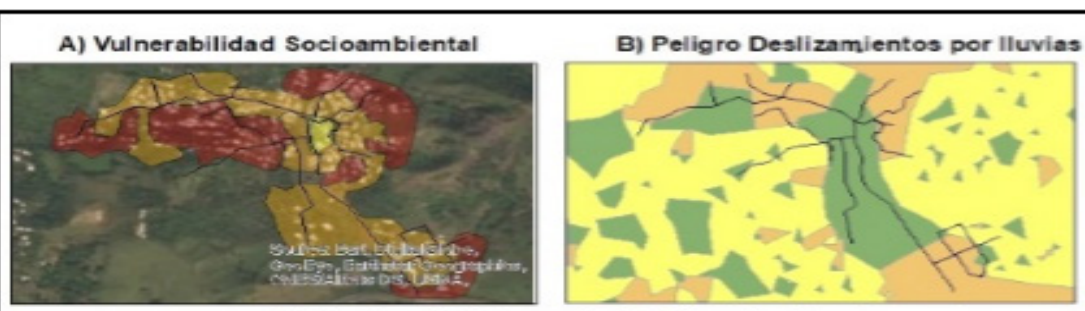

\section{C) Niveles de Riesgo de deslizamiento}
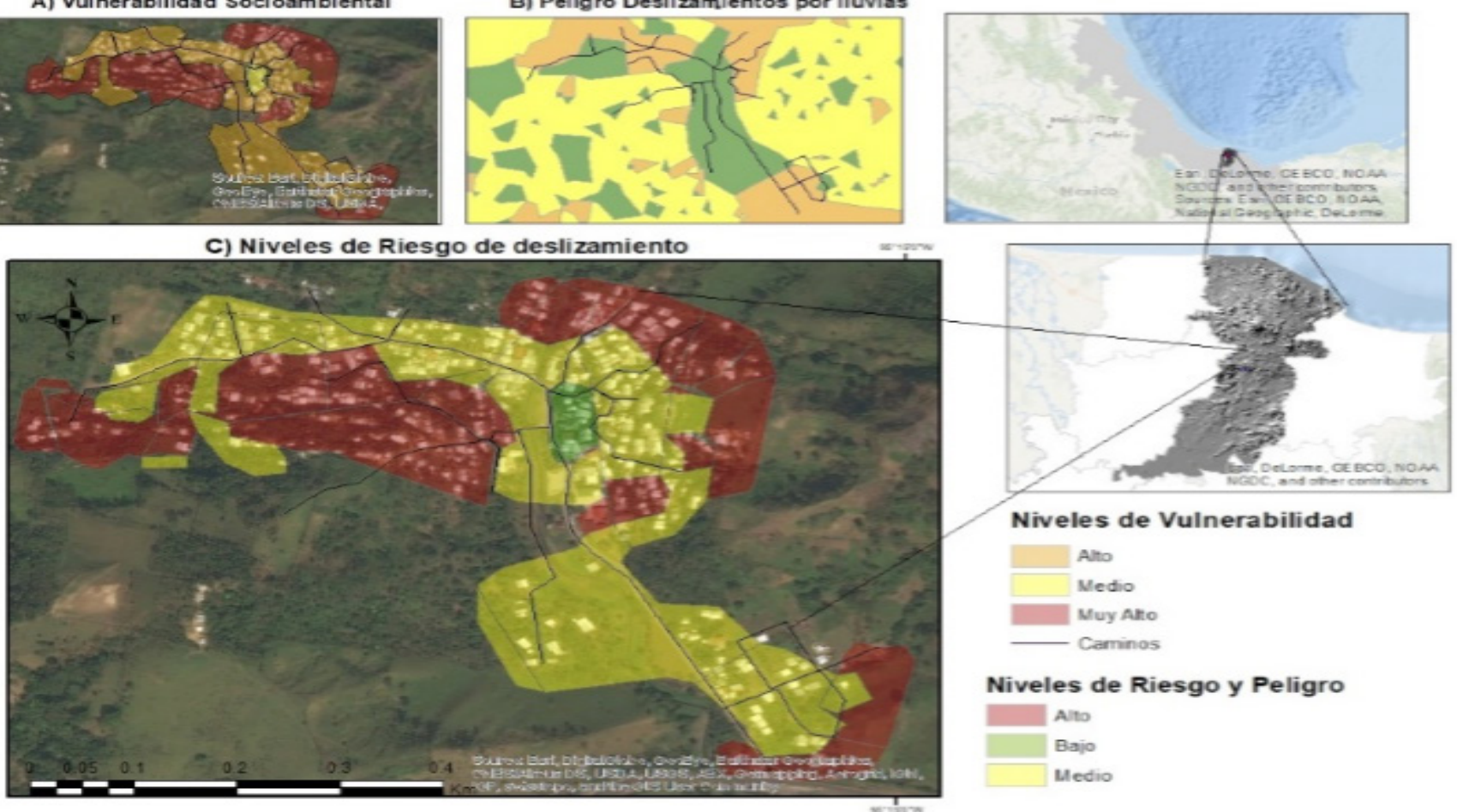

Niveles de Vulnerabilidad

Alino

$$
\begin{aligned}
& \text { Medio } \\
& \text { Muy Aro }
\end{aligned}
$$

- Caminos

Niveles de Riesgo y Peligro

Anto

Bajo

Medio 
nerabilidad media, 15 con vulnerabilidad alta y 11 muy alta, las viviendas con vulnerabilidad muy alta no existirían, 3 viviendas tendrían vulnerabilidad media y las demás tendrían vulnerabilidad alta. Lo mismo pasa con la variable de respuesta de la comunidad, la cual cuenta con dos valores 1 "No Apropiada y 2 "Apropiada". Al analizar a las 3 localidades se concluyó que la respuesta de las localidades tiene el valor 1 "no apropiado", ya que prefieren enfrentar los peligros y amenazas por su cuenta, haciendo caso omiso a las recomendaciones de Protección Civil. De los encuestados la mayoría respondió que no se desplazan a los albergues cuando ocurren, por ejemplo, fuertes lluvias. En el caso de Nacimiento de Xogapan, si la respuesta fuera la apropiada no existirán viviendas con vulnerabilidad muy alta, sino que habría sólo 3 viviendas con vulnerabilidad media y las demás con vulnerabilidad alta.

En un escenario en el que la existencia de planes de prevención tenga un valor 3 "Sufi- ciente" y una respuesta de la comunidad con valor 2 "Apropiada", en Nacimiento de Xogapan existirían: 10 viviendas con vulnerabilidad media y 18 con vulnerabilidad alta.

Debido a estos resultados, se propone un mayor acercamiento entre Protección Civil y la población para así lograr que los planes de prevención ya existentes se lleven a cabo, ya que este distanciamiento o desconfianza por parte de la población se ve reflejado en los altos niveles de vulnerabilidad actuales. En caso de lograr lo anterior, se podría obtener una mejor respuesta por parte de la localidad, disminuyendo los niveles de vulnerabilidad considerablemente; en las Tablas 7 a 9 se muestra como cambiarían los niveles de vulnerabilidad de cada una de las localidades con respuestas más favorables a las preguntas referentes a existencia de planes de prevención y respuesta de la comunidad:

Tabla 7: Comparación entre valores de planes de prevención

\begin{tabular}{|c|c|c|c|c|c|c|}
\hline \multirow{2}{*}{} & \multicolumn{3}{|c|}{ Planes de prevención (Incipiente) } & \multicolumn{3}{|c|}{ Planes de prevención (Suficiente) } \\
\cline { 2 - 7 } & Media & Alta & Muy Alta & Media & Alta & Muy Alta \\
\hline Texcaltitán & 2 & 31 & 24 & 4 & 52 & 1 \\
\hline Tonalapan & 3 & 16 & 13 & 6 & 25 & 1 \\
\hline $\begin{array}{c}\text { Nacimientos de } \\
\text { Xogapan }\end{array}$ & 2 & 15 & 11 & 3 & 25 & 0 \\
\hline
\end{tabular}

Tabla 8: Comparación entre valores de respuesta de la localidad

\begin{tabular}{|c|c|c|c|c|c|c|}
\hline \multirow{2}{*}{} & \multicolumn{3}{|c|}{$\begin{array}{c}\text { Respuesta de la localidad } \\
\text { (No Apropiada) }\end{array}$} & \multicolumn{3}{c|}{$\begin{array}{c}\text { Respuesta de la localidad } \\
\text { (Apropiada) }\end{array}$} \\
\cline { 2 - 7 } & Media & Alta & Muy Alta & Media & Alta & Muy Alta \\
\hline Texcaltitán & 2 & 31 & 24 & 4 & 52 & 1 \\
\hline Tonalapan & 3 & 16 & 13 & 6 & 25 & 1 \\
\hline $\begin{array}{c}\text { Nacimientos de } \\
\text { Xogapan }\end{array}$ & 2 & 15 & 11 & 3 & 25 & 0 \\
\hline
\end{tabular}


Tabla 9: Comparación entre valores de planes de prevención y respuesta de la localidad

\begin{tabular}{|c|c|c|c|c|c|c|}
\hline & \multicolumn{3}{|c|}{$\begin{array}{c}\text { Planes de prevención (Incipiente) y } \\
\text { Respuesta de la localidad (No Apro- } \\
\text { piada) }\end{array}$} & $\begin{array}{c}\text { Planes de prevención (Suficien- } \\
\text { te) y Respuesta de la localidad } \\
\text { (Apropiada) }\end{array}$ \\
\cline { 2 - 7 } & Media & Alta & Muy Alta & Media & Alta & Muy Alta \\
\hline Texcaltitán & 2 & 31 & 24 & 7 & 50 & 0 \\
\hline Tonalapan & 3 & 16 & 13 & 8 & 24 & 0 \\
\hline $\begin{array}{c}\text { Nacimiento de } \\
\text { Xogapan }\end{array}$ & 2 & 15 & 11 & 10 & 18 & 0 \\
\hline
\end{tabular}

Propuesta de medidas de mitigación de peligro y riesgo

Las tres localidades analizadas en este estudio enfrentan peligros por fenómenos naturales de diversa índole: Los Tuxtlas es una zona con volcanismo activo y entre los fenómenos que más se espera, ocurran durante una futura actividad es la caída de ceniza y flujos de lava. Aparte de contaminar cuerpos de agua y afectar actividades cotidianas y la salud de vías respiratorias, la ceniza se acumula en los techos de las construcciones humanas, causando su colapso en el caso de sobrepasar cierta carga (dependiendo del tipo de techo). En el área de estudio el peligro de colapso de techos por caída de ceniza sólo alcanza el nivel medio en $\mathrm{Na-}$ cimiento de Xogapan; en el caso de Texcaltitán no existe el peligro mientras que en Tonalápan el nivel es bajo.

Mientras tanto los deslizamientos y procesos de remoción en masa, son comunes en Los Tuxtlas ya que son ocasionados por las precipitaciones elevadas y topografía accidentada presente en la zona, en las 3 localidades estudiadas se presentan niveles de peligro alto, medio y bajo. De acuerdo a la matriz de INDECI (2006) se propone lo siguiente:

Peligro bajo: Estas zonas tienen menos probabilidades de ser afectados por fenómenos naturales por lo que se prestan para expansión urbana y establecimiento de equipamientos.

Peligro medio: En este caso existe la probabi- lidad de ser afectados por fenómenos naturales por lo que se recomienda expansión urbana, pero con materiales adecuados.

Peligro alto: Estas zonas están susceptibles a los efectos de los fenómenos naturales por lo que, si existen viviendas, éstas deben de tener materiales y sistemas de construcción adecuados; tampoco está permitida la alta densidad de viviendas.

En lo que se refiere al riesgo en las 3 localidades existen zonas de alto, medio y bajo riesgo, se propone lo siguiente:

Riesgo bajo: Estas zonas son las más recomendables para el establecimiento de grandes equipamientos, por ejemplo escuelas, hospitales o cuarteles de policía.

Riesgo medio: Los habitantes de estas zonas necesitan ser informados sobre los peligros y amenazas de la zona, además de capacitación en medidas de prevención; para el caso de nuevas viviendas lo recomendable es que se hagan mediante restricciones (distancia adecuada al río, baja pendiente, distancia adecuada a zonas de deslizamiento, techos y estructuras resistentes).

Riesgo alto: Mantener informados a los habitantes de estas zonas sobre los peligros y amenazas, capacitación en medidas de prevención de los habitantes y autoridades, se recomienda una baja densidad de viviendas, ni tampoco el establecimiento de escuelas u hospitales, para el establecimiento de nuevas viviendas se recomienda limitarlas o exigir estructuras y adecuaciones resistentes 


\section{Conclusiones}

Los estudios de vulnerabilidad son un tema que se ha abordado de varias formas, social, ambiental, política, etc., pero a pesar de eso, es difícil encontrar metodologías que sean replicables. Con las metodologías de Bohórquez (2013) y Lozano (2008) se da un panorama más claro para evaluar la vulnerabilidad y el riesgo, al prestarse para ser aplicadas para estudios futuros. Con la primera metodología se reconocieron una mayor cantidad de variables, demostrando que no sólo la estructura de la vivienda es la responsable de la vulnerabilidad (física), sino también las políticas públicas, el acceso a la información y la oferta de servicios. Se pudo observar que incluso las viviendas con buenas condiciones estructurales también pueden tener niveles altos de vulnerabilidad, debido a la informalidad de sus habitantes y de la misma localidad. Obtenida la vulnerabilidad de las localidades y complementándola con la segunda metodología, se obtuvieron los mapas de riesgo. A continuación, utilizando la matriz elaborada por (INDECI, 2006) del estudio de Lozano (2008) se realizan las siguientes propuestas.

\section{Referencias}

Bohórquez, J. (2013). Evaluación de la vulnerabilidad social ante amenazas naturales en Manzanillo. Investigaciones geográficas. Instituto de Geografía. Universidad Nacional Autónoma de México, México, 79-93.

Bollin, C.; Hidajat, R. (2006). Community-based risk index: pilot implementation in Indonesia. En: Birkmann, J. (ed) Measuring Vulnerability to natural hazards. Towards disasters resilient societies, United Nations University Press, Tokio, Japon, 271-289.

Chardon, A; González, J. (2002). Amenaza, vulnerabilidad, riesgo, desastre, mitigación, prevención. En: Primer acercamiento, características y metodologías de análisis y evaluación. Instituto de estudios ambientales, Universidad Nacional de Colombia, Colombia, 38 p.

Hernández, L.; Cruz, H.; Márquez, B.; Suarez, C.; Padlog, M.; Palomar, P. (2003). Aproximación al análisis de la vulnerabilidad del Volcán de Colima (Jalisco, Mexico), Vegueta, México, 241-254.
Instituto Nacional de Defensa Civil (INDECI) (2006). Manual Básico para la estimación del riesgo. Lima, Republica del Perú, 69 p.

Instituto Nacional de Estadística e Informática (INEGI) (2010). Prontuario de Información Geográfica Municipal de San Andrés Tuxtla. México.

Lara, L.; Clavijero, J.; Hinojosa, M.; Huerta, S.; Wall, R.; Moreno, H. (2006). NVEWS-Chile: Sistema de clasificación semi-cualitativa de la vulnerabilidad volcánica. Universidad Tecnológica Metropolitana, Chile.

Lozano, O. (2008). Metodología para el análisis de vulnerabilidad y riesgo ante inundaciones y sismos, de las edificaciones en centros urbanos. Centro de estudios y prevención de desastres, Perú.

Reyes, C.; Flores, L.; Pacheco, M.; López, O.; Valerio, L.; Zepeda, R. (2005). Evaluación de la Vulnerabilidad. Capitulo VIII. Evaluación simplificada de la vulnerabilidad de la vivienda unifamiliar ante sismo y viento. Centro Nacional para la Prevención de Desastres, México, 311-338.

Sieron, K.; Capra, L; Rodríguez, S.R. (2014). Hazard assessment at San Martín volcano based on geological record, numerical modeling and spatial analysis. Natural Hazards, 70, 1, 275-297, doi. 10.1007/s11069-013-08077.

Wisner, B.; Holling. S.; Carpenter S.; Kinzig A. (2004). At risk: natural hazards, people's vulnerability and disasters. Routledge. Londres. Inglaterra. En: Ruiz, N. 2012. La definición y medición de la vulnerabilidad social. Un enfoque normativo. Investigaciones geográficas. Instituto de Geografía UNAM. México, 63-74. 